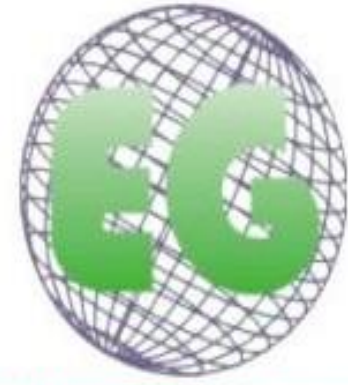

ISSN 1695-6141 $N^{\circ} 45$

\title{
Efectividad del tratamiento tópico de la flebitis secundaria a la cateterización periférica: una revisión sistemática
}

Efectiveness of topical therapies for phlebitis related to peripheral intravenous canulation: a sistematic review

\section{*Martín Gil, Belén *Fernández Castro, Mercedes ** López Vallecillo, Maria **Peña García, Isabel}

\author{
*Enfermera de la Unidad de Oncología Radioterápica y Medicina Nuclear del Hospital Clínico \\ Universitario de Valladolid. E-mail: bmartingi@saludcastillayleon.es **Enfermera de la Unidad de \\ Gestión de Cuidados GACELA CARE del Hospital Clínico Universitario de Valladolid. España,
}

http://dx.doi.org/10.6018/eglobal.16.1.260411

\section{RESUMEN}

Introducción: La flebitis es la complicación más frecuente secundaria a la cateterización periférica.

Objetivo: Evaluar la evidencia disponible sobre la efectividad de tratamientos tópicos de la flebitis postperfusión en pacientes portadores de catéter venoso periférico.

Método: Se realizó revisión sistemática de ensayos clínicos y revisiones sistemáticas publicados desde 1995 a 2015 en las bases de datos Pubmed, Cinahl, Cochrane plus CUIDEN y la BVS (Biblioteca Virtual en Salud), en inglés y español. Los criterios de inclusión fueron: paciente adulto hospitalizado, portador de vía venosa periférica, con perfusión continua o intermitente, incluyendo todo tipo de terapia de infusión que hubieran desarrollado cualquier grado de flebitis. La valoración de la calidad metodológica de los estudios seleccionados, se realizó por pares de forma independiente utilizando el Clinical Appraisal Skills Programme en su versión española.

Resultados: Se seleccionaron 11 estudios: 8 ensayos clínicos y 3 revisiones sistemáticas. Analizaron los efectos de fitoterapéuticos: aloe vera, chamomilla recutita y notoginseny; pomadas heparinoides y geles de heparina; antiinflamatorios como el diclofenaco y vasodilatadores como la nitroglicerina, resultando todos ellos efectivos.

Conclusiones: El aloe vera, notoginseny, diclofenaco y heparina gel 1000 UI, mostraron nivel de evidencia y grado de recomendación moderado. La heparina gel es el único compuesto con indicación de la Agencia Española del Medicamento para tratar la flebitis post-perfusión, el notoginseny no está comercializado en Occidente y el diclofenaco es un antiinflamatorio utilizado en diversas patologías. Es merecedor de especial atención el tratamiento con aloe vera a la espera de estudios más concluyentes. 
Palabras clave: Flebitis; tratamiento; catéter periférico; infusión intravenosa

\section{ABSTRACT}

Background: Phlebitis is the most common complication induced by peripheral intravenous catheter.

Aim: To assess the best available evidence concerning the effectiveness of topical therapies in patients with peripheral venous canulation who developed phlebitis

Method: The bibliographic search for clinical trials and sistematic reviews, published between 1995 and 2015, was carried out in the following databases Pubmed, Cinahl, Cochrane plus CUIDEN and BVS (Biblioteca Virtual en Salud), in english and spanish. Inclusion criteria were: adult inpatient with peripheral venous cateterization with continous or intermitent infusion, including all types of infusion therapy who developed any degree of superficial phlebitis. The quality of selected studies was assessed independently by peer reviewers using the Clinical Appraisal Skills Programme in its spanish version.

Results: 11 studies were identified, 8 clinical trials and 3 sistematic reviews. The efectiveness of topical phytotherapeutics: aloe vera, chamomilla recutita and notoginseny; heparinoid creams and heparin gels; anti-inflamatory as diclofenac, and vasodilators as nitroglycerin was analized, showing effectivity all of them.

Conclusions: Aloe vera, notoginseny, diclofenac and heparine gel suggest a weak level of evidence and moderate grade of recommendation. Heparin gel is the only product provided by the Spanish Medicine Agency to treat post-infusion phlebitis, notoginseny is not marketed in Spain and diclofenac is an anti-inflammatory used for various pathologies. Deserves special attention of topical therapy with aloe vera but further studies are needed.

Keywords: Phlebitis; treatment; peripheral catéter; intravenous infusion

\section{INTRODUCCIÓN}

La canalización de la vía venosa es una de las técnicas más practicada por el personal de enfermería en los hospitales ${ }^{(1,2)}$, ya que uno de cada tres pacientes es portador de un catéter venoso periférico $(\mathrm{CVP})^{(3)}$, siendo la flebitis la complicación más común ${ }^{(1,4-6)}$.

Se define flebitis como la inflamación de la vena en el tramo o segmento tunelizado por un catéter, cuyo origen tiene distintas causas. Éstas pueden ser: químicas por perfusión de medicación irritante, mecánicas debidas al lugar de punción, tipo y fijación del catéter e infecciosas causadas por la colonización de agentes patógenos cuyo origen es la piel y que migran hacia el interior ${ }^{(5-8)}$.

Se debe realizar un diagnóstico diferencial con la tromboflebitis y la extravasación, aunque estos dos efectos adversos suelen aparecer asociados a la misma por la propia fisiopatogenia de la flebitis. El proceso inflamatorio se inicia con la sensibilización del endotelio vascular debido a la fricción en la pared de la vena por el propio catéter, hiperosmolaridad de la solución administrada o las toxinas bacterianas. Esto conduce a la liberación de serotonina e histamina produciendo vasodilatación y el consiguiente edema. A la vez, la histamina favorece la agregación plaquetaria formándose un cordón trombótico palpable que con la evolución del proceso se hará visible a lo largo de la vena canalizada, apareciendo calor localizado ${ }^{(5)}$. Los signos y síntomas característicos de la flebitis son: eritema, dolor, calor local, y edema, también pueden aparecer otros como: cordón indurado a la palpación, exudado purulento y fiebre ${ }^{(7)}$. 
Se describen cuatro grados de flebitis en función de la gravedad de los signos y síntomas clínicos, que van de 0 a 4, siendo 0 la ausencia de síntomas, 1 eritema con o sin dolor, 2 dolor con eritema o edema o ambos, 3 dolor, eritema o edema y cordón fibroso palpable, y 4 cuando están presentes todos los síntomas: dolor en el punto de acceso con eritema o edema o ambos, cordón venoso palpable de $2,5 \mathrm{~cm}$ de longitud y secreción purulenta ${ }^{(5-8)}$.

En los pacientes con CVP, el riesgo de flebitis se incrementa con el número de días de cateterización y la concurrencia de otros factores como la edad avanzada, el sexo femenino, la localización de punción o la administración de algunos fármacos (amoxicilina-clavulánico y aminoglucósidos) ${ }^{(2)}$.

La incidencia de la flebitis varía dependiendo del tiempo de seguimiento, las unidades asistenciales y la definición de la misma. Hay que destacar la variabilidad en los estudios, que muestran una incidencia de flebitis del $0.5 \%$ al $59,1 \%$ entre los pacientes que reciben terapia intravenosa periférica ${ }^{(1)}$. Algunos autores manejan datos de prevalencia del $2.5 \%-70 \%$ con medias porcentuales del $25-35 \%{ }^{(4)}$.

Los signos y síntomas de la flebitis producen disconfort en el paciente, requieren en ocasiones la canalización de una nueva vía, siendo difícil, en casos de malos accesos o repetidas situaciones de flebitis. Para poder dar continuidad al tratamiento ${ }^{(9)}$ puede ser necesaria la inserción de una vía venosa central, esto repercute en el gasto sanitario, tanto por el incremento de recursos materiales como por el aumento del tiempo que el equipo de enfermería invierte en recanalizar otra vía venosa, reinstaurar el tratamiento y proveer atención y cuidados al paciente afectado ${ }^{(8)}$.

La flebitis asociada a los dispositivos venosos causa infección nosocomial, lo que puede suponer una prolongación de la estancia hospitalaria e incluso complicarse con una sepsis, aunque la incidencia es baja ${ }^{(10)}$. En la mayoría de los casos se trata de infección por Estafilococo Aureus que puede desembocar en una bacteriemia ${ }^{(9)}$ con una mortalidad anual considerable debido a la gran utilización de CVP ${ }^{10}$.

El abordaje y cuidado de los pacientes con terapia intravenosa forma parte del trabajo asistencial de las enfermeras ${ }^{(11)}$. Cabe destacar que una de las actividades de la intervención NIC "Terapia intravenosa" consiste en "Observar si se presentan signos y síntomas asociados a la flebitis por la infusión e infecciones locales" ${ }^{(12)}$ relacionada con la detección precoz de la misma. Cuando aparece alguno de los síntomas, generalmente, el tratamiento consiste en la interrupción de la infusión y retirada de la vía, para posteriormente aplicar compresas templadas o frías ${ }^{(13)}$ y dependiendo del grado de flebitis, puede requerir intervención médica ${ }^{(14)}$.

En la práctica clínica existe una gran variabilidad en el tratamiento tópico de la flebitis, que tradicionalmente se ha basado en la aplicación de calor y analgesia, siendo los antiinflamatorios beneficiosos para reducir la inflamación en el punto de inserción ${ }^{(15)}$. También hay constancia de estudios sobre la posible eficacia de los corticoides y la heparina, solos o en combinación, así como de la nitroglicerina tópica (NTG) ${ }^{(16)}$. La Guía de Práctica Clínica sobre terapia intravenosa con dispositivos no permanentes en adultos, publicada por el Ministerio de Sanidad, Servicios Sociales e Igualdad de 2014, no establece recomendaciones para el tratamiento de la flebitis secundaria a catéteres venosos, pero sí sugiere este tema entre las futuras líneas de investigación $^{(17)}$. Es complejo describir cuál sería el tratamiento de elección de la 
flebitis secundaria a la inserción de una vía periférica. Por ello, se plantea la búsqueda de la mejor evidencia disponible en la literatura médica o científica.

\section{OBJETIVO}

Valorar la efectividad de los tratamientos tópicos de la flebitis secundaria a la cateterización venosa periférica, aplicados a pacientes hospitalizados, en relación a la mejoría o resolución de los signos y síntomas. Como objetivo secundario: identificar, dentro de los tratamientos eficaces, cuáles serían las características de su aplicación: frecuencia, dosis, etc.

\section{MÉTODO}

Diseño: Se realizó una revisión sistemática de ensayos clínicos (EC) y revisiones sistemáticas (RS), en busca de la mejor evidencia en el tratamiento tópico de la flebitis relacionada con acceso venoso periférico.

Criterios de inclusión: Paciente adulto hospitalizado, portador de vía venosa periférica tanto con perfusión continua como intermitente, incluyendo todo tipo de terapia de infusión y que hubieran desarrollado cualquier grado de flebitis en miembro superior. Respecto a la intervención se contemplan los tratamientos tópicos en la zona afectada que tuvieran como resultados el dolor medido a través de cualquier escala y el eritema en centímetros (cm), además del calor, edema, induración, cordón fibroso y tiempo de resolución de los signos y síntomas. Se excluyeron los ensayos clínicos que trataban pacientes no hospitalizados, pediátricos exclusivamente y aquellos que fueran portadores de vías venosas centrales, incluidas las de inserción periférica, además de los tratamientos de la flebitis por vía oral o parenteral.

Búsqueda bibliográfica: Se realizó una búsqueda de EC y RS publicados desde 1995 a 2015, en inglés y en español, en las bases de datos Pubmed, Cinahl, Cochrane plus CUIDEN y la BVS (Biblioteca Virtual de Salud). Para las tres primeras se utilizaron descriptores Mesh con las siguientes ecuaciones de búsqueda: [("catheterization peripheral" OR "infusion, intravenous") AND "phlebitis"], ["Phlebitis" AND "nursing"], ["Thrombophlebitis" AND "therapy" AND "arm"], ["Phlebitis" AND "Pain"]; así como las frases en texto libre: ["infusion intravenous phlebitis and management nursing care"], ["topical treatment and infusion therapy phlebitis"], ["topical treatment and phlebitis"], ["Infusion intravenous phlebitis and management"], ["peripheral phebitis nursing therapy"], ["Treatment phlebitis infusion"], [Phlebitis and Treatment outcomes]. Para las bases de datos de CUIDEN y BVS se utilizaron los descriptores DeCS "flebitis" y "tratamiento". Además, se han consultado TESEO y GUÍA SALUD y las páginas web: Infusion Nurses Society (INS) y Asociación de Enfermería de Equipos de Terapia Intravenosa (ETI).

La pregunta formato PICO planteada fue: "Efectividad de los tratamientos tópicos en pacientes hospitalizados con flebitis secundaria a cataterización venosa periférica para mejorar o resolver sus signos y síntomas".

Cuatro investigadoras realizaron la búsqueda para extraer referencias bibliográficas de forma independiente. Se examinaron los títulos y los resúmenes de los artículos para identificar su viabilidad, optando por aquellos que cumplían los criterios de inclusión establecidos. La última búsqueda se llevó a cabo el 30 de noviembre de 2015. 
Selección de estudios y extracción de datos: Una vez puestos en común los hallazgos no hubo discrepancias en la elección de estudios a texto completo. Para la lectura crítica dos enfermeras analizaron los EC y otras dos las RS, en ambos casos por pares, resolviendo las discrepancias por consenso. El instrumento utilizado para la valoración de la calidad de los estudios fue la Guía de Valoración Crítica de Ensayos Clínicos Aleatorios y Guía de Valoración Crítica de Revisiones Sistemáticas del Critical Appraisal Skills Program en español $(\mathrm{CASPe})^{(18)}$, acordando entre los cuatro investigadores incluir los estudios con CASPe igual o superior a 7 sobre 11 items para los EC (Tabla I) y 6 sobre 10 para las RS (Tabla II), tratando de asegurar una buena calidad metodológica y bajo riesgo de sesgo. Para la extracción de datos, se diseñó una tabla en la que se incluyó año de publicación, país, tipo de estudio, características de la muestra, intervención, variables estudiadas, resultados y comentarios adicionales como el nivel de evidencia y grado de recomendación según los criterios de Joanna Briggs Institute ${ }^{19}$ (Tabla III). Debido a la heterogeneidad de los resultados, estos se presentan mediante un resumen narrativo de las intervenciones estudiadas, para dar respuesta a la pregunta de búsqueda.

\section{RESULTADOS}

Descripción de hallazgos: La búsqueda estructurada en Pubmed identificó 1077 referencias, en CINAHL 179, en Cochrane 163 y BVS (Lilacs, IBECS) 103, Cuiden 36, lo que permitió identificar un total de 1558. Eliminando duplicados quedaron 1397 estudios. Tras el análisis del título y resumen se obtuvieron 33 artículos a texto completo, de los que se excluyeron 22 por centrarse en la prevención y no ser revisiones sistemáticas, ni ensayos clínicos. Se evaluaron metodológicamente 11 artículos de los cuales, 8 fueron EC ${ }^{(20-27)}$ y $3 \mathrm{RS}^{(28-30)}$. (Figura 1). 
Fig.1- Diagrama de flujo para la selección de artículos

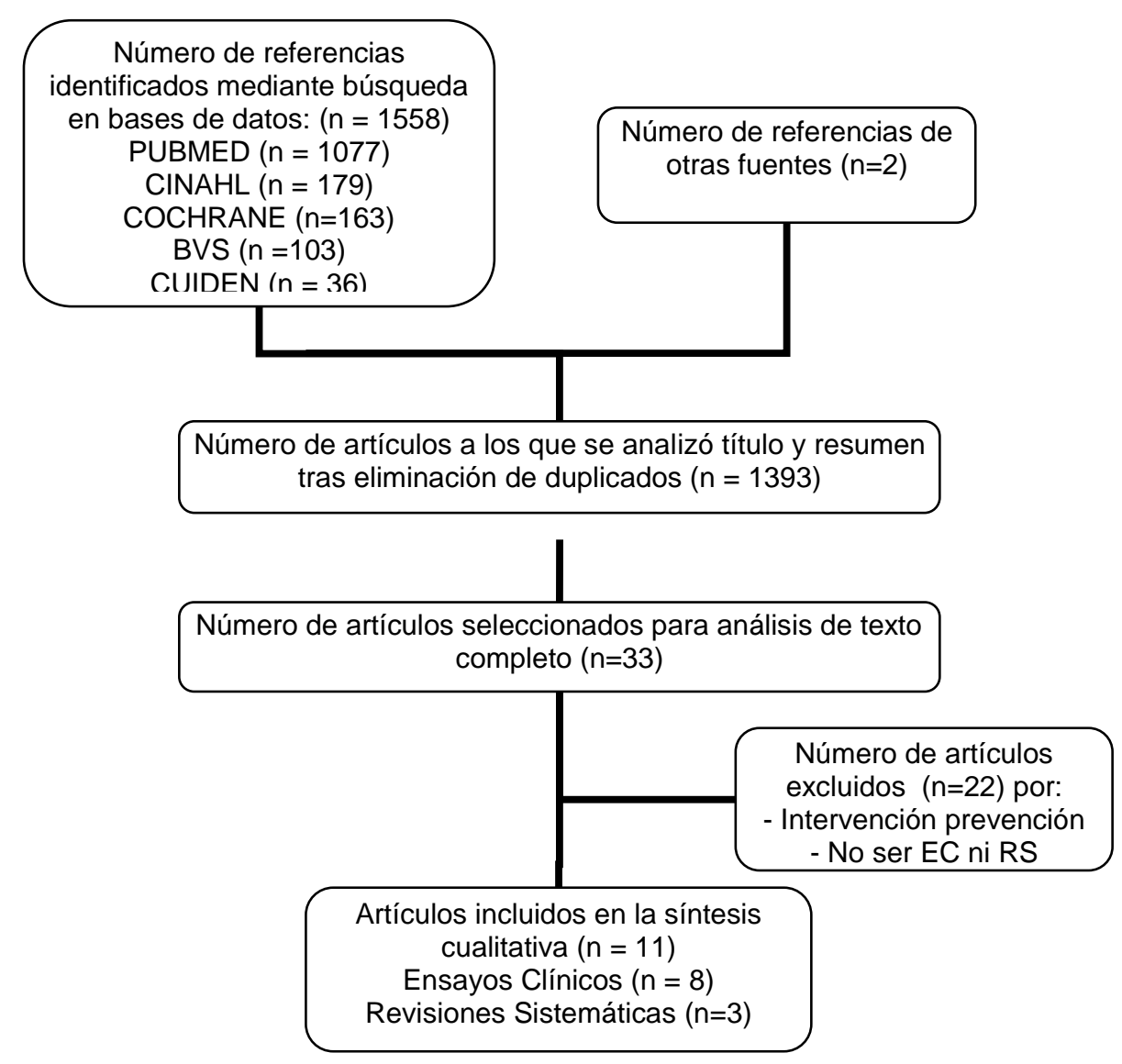

Calidad metodológica y características de los estudios: Los estudios revisados presentan variabilidad en cuanto a calidad metodológica. La puntuación media de CASPe para los EC fue de 8,625, y para las RS fue de 8,66. Ver tablas de valoración de CASPe (Tablas I y II).

Hay que reseñar deficiencias de calidad metodológica en dos estudios ${ }^{(20,27)}$ en los que la asignación de los pacientes a los grupos de intervención es cuasi-aleatoria y en tres $(20,24,25)$ en los que el tamaño muestral es pequeño. Dos están mejor diseñados que el resto ${ }^{(22,23)}$ y solo dos son doble ciego ${ }^{(23,24)}$. En todos los estudios los grupos fueron homogéneos al comienzo del ensayo, se trataron de igual forma y el seguimiento fue completo teniendo en cuenta los resultados relevantes. En cuanto a la valoración del efecto, presentan medidas de asociación o efecto (RR, OR, HR) $>1$ dos ${ }^{(22,23)}$ de los 8 ensayos, en los restantes no ha sido calculado. Los resultados son precisos en tres de ellos ${ }^{(22,23,26)}$, mostrando un IC $>95 \%$, en los demás no se aclara la precisión de los mismos (Tabla I).

En cuanto a las revisiones, la puntuación CASPe varía entre los 10 puntos de Zheng et $\mathrm{al}^{(28)}$ y los 7 de Dos Reis et $\mathrm{al}^{(29)}$ (Tabla II). En todas ellas se incluyeron estudios pertinentes o relevantes, aunque el esfuerzo por valorar la calidad metodológica de los mismos varía. Así mismo todas extraen un resultado global y consideran los resultados importantes. En una revisión ${ }^{(28)}$ debido al mayor número de estudios, los resultados se han combinado estadísticamente siendo más precisa que el resto. 
En referencia al idioma utilizado en los EC el 50\% de los estudios están escritos en inglés $^{(21,23,24,26)}$ y el otro $50 \%$ en español ${ }^{(20,22,25,27)}$. Las revisiones ${ }^{(28-30)}$ son las tres en inglés.

Tabla I.- Análisis de lectura crítica para evaluación de EC - CASPe

\begin{tabular}{|c|c|c|c|c|c|c|c|c|}
\hline & $\begin{array}{l}\text { Reis } \\
\text { PE et } \\
\text { al. }\end{array}$ & $\begin{array}{l}\text { Gouping } \\
\text { Z et al. }\end{array}$ & $\begin{array}{l}\text { Becheru- } \\
\text { cci A } \\
\text { et al. }\end{array}$ & $\begin{array}{l}\text { Vilardell } \\
\text { M et al. }\end{array}$ & $\begin{array}{l}\text { De } \\
\text { Sanctis } \\
\text { MT et } \\
\text { al. }\end{array}$ & $\begin{array}{l}\text { Ruiz } \\
\text { Trillo } \\
\text { Cet al. }\end{array}$ & $\begin{array}{l}\text { IHL } \\
\text { NG et } \\
\text { al. }\end{array}$ & $\begin{array}{l}\text { Córcoles } \\
\text { MP } \\
\text { et } a l .\end{array}$ \\
\hline $\begin{array}{l}\text { Pregunta bien } \\
\text { definida }\end{array}$ & $\mathrm{Si}$ & $\mathrm{Si}$ & $\mathrm{Si}$ & $\mathrm{Si}$ & $\mathrm{Si}$ & $\mathrm{Si}$ & $\mathrm{Si}$ & $\mathrm{Si}$ \\
\hline Aleatorización & $\mathrm{Si}$ & $\mathrm{Si}$ & $\mathrm{Si}$ & $\mathrm{Si}$ & $\mathrm{Si}$ & $\mathrm{Si}$ & $\mathrm{Si}$ & $\mathrm{Si}$ \\
\hline $\begin{array}{l}\text { Seguimiento } \\
\text { completo }\end{array}$ & $\mathrm{Si}$ & $\mathrm{Si}$ & $\mathrm{Si}$ & $\mathrm{Si}$ & $\mathrm{Si}$ & $\mathrm{Si}$ & $\mathrm{Si}$ & $\mathrm{Si}$ \\
\hline Doble ciego & no & ¿? & ¿? & $\mathrm{Si}$ & $\mathrm{Si}$ & No & no & no \\
\hline $\begin{array}{l}\text { Homogeneidad } \\
\text { de los grupos }\end{array}$ & si & $\mathrm{Si}$ & $\mathrm{Si}$ & $\mathrm{Si}$ & $\mathrm{Si}$ & $\mathrm{Si}$ & $\mathrm{Si}$ & $\mathrm{Si}$ \\
\hline $\begin{array}{l}\text { Los grupos } \\
\text { fueron tratados } \\
\text { de igual modo }\end{array}$ & si & $\mathrm{Si}$ & $\mathrm{Si}$ & $\mathrm{Si}$ & $\mathrm{Si}$ & $\mathrm{Si}$ & $\mathrm{Si}$ & $\mathrm{Si}$ \\
\hline $\begin{array}{l}\text { Es grande el } \\
\text { efecto del } \\
\text { tratamiento }\end{array}$ & $i ?$ & ¿? & $\mathrm{Si}$ & $\mathrm{Si}$ & ¿? & ¿? & $i ?$ & $\mathrm{Si}$ \\
\hline $\begin{array}{l}\text { Son precisos } \\
\text { los resultados } \\
\text { IC } \geq 95 \%\end{array}$ & $i ?$ & $i ?$ & $\mathrm{Si}$ & $\mathrm{Si}$ & $i ?$ & $i ?$ & $\mathrm{Si}$ & $i ?$ \\
\hline $\begin{array}{l}\text { Son aplicables } \\
\text { los resultados }\end{array}$ & $i ?$ & $\mathrm{Si}$ & $\mathrm{Si}$ & $\mathrm{Si}$ & $\mathrm{Si}$ & $i ?$ & $\mathrm{Si}$ & $i ?$ \\
\hline $\begin{array}{l}\text { Se tuvieron en } \\
\text { cuenta todos } \\
\text { los resultados } \\
\text { importantes }\end{array}$ & $\mathrm{Si}$ & $\mathrm{Si}$ & $\mathrm{Si}$ & si & $\mathrm{Si}$ & si & $\mathrm{Si}$ & $\mathrm{Si}$ \\
\hline Total & 7 & 8 & 10 & 11 & 9 & 7 & 9 & 8 \\
\hline
\end{tabular}

Tabla II.- Análisis de lectura crítica para evaluación de RS - CASPe

\begin{tabular}{|l|l|l|l|}
\hline & $\begin{array}{l}\text { Zheng } \\
\text { G H } \\
\text { et al. }\end{array}$ & $\begin{array}{l}\text { Dos } \\
\text { Reis } \mathbf{P} \text { et al. }\end{array}$ & $\begin{array}{l}\text { Di } \\
\text { Nisio } \\
\text { et al. }\end{array}$ \\
\hline Tema claramente definido & si & si & si \\
\hline Artículos adecuado & si & si & si \\
\hline Incluidos los estudios importantes y pertinentes & si & si & si \\
\hline Suficiente esfuerzo para valorar la calidad de los estudios incluidos & si & no & si \\
\hline Resultados de los diferentes EC mezclados para resultado combinado & si & no & si \\
\hline ¿Cuál es el resultado global de la revisión? & si & si & si \\
\hline ¿Cuál es la precisión del resultado? & si & no & ¿? \\
\hline ¿Se pueden aplicar los resultados en tu medio? & si & si & $\mathrm{Si}$ \\
\hline $\begin{array}{l}\text { ¿Se consideraron todos los resultados importantes para tomar } \\
\text { decisión? }\end{array}$ & si & si & $\mathrm{Si}$ \\
\hline ¿Los beneficios merecen la pena frente a los perjuicios y costes? & si & si & $\mathrm{Si}$ \\
\hline Total & $\mathbf{1 0}$ & $\mathbf{7}$ & $\mathbf{9}$ \\
\hline
\end{tabular}


Características de la población: El número total de pacientes estudiados en los EC es de 545. La flebitis se determina a través de las escalas de la Infussion Nursing Society of América (INS) ${ }^{(20,21,23)}$ y de Maddox ${ }^{(25)}$, en el resto a través de los signos y síntomas. Los participantes tratados en los diferentes estudios son mayores de 18 años, exceptuando en uno ${ }^{(21)}$ cuya edad varía de 4 a 90 años. Se les administra cualquier tipo de medicación intravenosa (antibioterapia, sueroterapia, soluciones hipertónicas, manitol, derivados sanguineos etc.) exceptuando en un $\mathrm{EC}^{(20)}$, en que la medicación es únicamente quimioterápica. Dichos pacientes se encuentran hospitalizados en plantas de Oncología ${ }^{(20)}$. Medicina Interna ${ }^{(22,23,25)}$ y Cirugía General ${ }^{(27)}$, en los demás estudios no se menciona el lugar de hospitalización.

De los 133 estudios que examinan las tres $\mathrm{RS}^{(28-30)}$ analizadas, únicamente 36 abordan el tratamiento tópico de la flebitis relacionado con la vía venosa periférica, con un total de 3134 pacientes. El diagnóstico de flebitis en los estudios de la revisión de Zheng GH et al $^{(28)}$ se realizó utilizando la escala de INS o la Nursing Conference Hangzhou City. En las otras dos revisiones ${ }^{(29,30)}$ se realiza a través de los signos clínicos. Una gran mayoría de la población de los estudios de la revisión de Zheng GH et $\mathrm{al}^{(28)}$ son pacientes oncológicos en tratamiento con quimioterapia.

Análisis de los datos: Respecto a las variables de intervención estudiadas tanto en las RS como en los EC, se analiza la efectividad de los tratamientos tópicos a través de los signos y síntomas de la flebitis y su evolución. Las variables estimadas son el eritema, tiempo de resolución del mismo ${ }^{(20,21,23,26,27)}$, el calor ${ }^{(22,24,27)}$, dolor ${ }^{(22,24,27)}$ y la inflamación ${ }^{(22-25)}$. La mayoría de los trabajos valoraron los efectos adversos y la toxicidad secundaria al tratamiento estudiado ${ }^{(20-25)}$. Se tuvieron en cuenta además otras variables dependientes como localización ${ }^{(21-23,25,27)}$, tipo de catéter ${ }^{(21-23,27)}$, tipo de infusión ${ }^{(20-23,25,27)}$ e incidencia de la flebitis ${ }^{(21-23,25,27)}$. Las tres revisiones ${ }^{(28-30)}$ también evalúan los signos y síntomas de la flebitis y todas tienen como objetivo primario la tasa de resolución de la flebitis, el tiempo de regresión y la disminución de los signos o síntomas como el eritema, dolor o edema.

A continuación se presenta un breve resumen descriptivo sobre la efectividad de las intervenciones estudiadas, cuyos datos se encuentran detallados en la Tabla III. Se ha dividido en cuatro grandes grupos para darle un sentido integrador.

\section{Efecto de los fitoterapéuticos:}

Chamomilla recutita al $1 \%$ fente al 2,5\% frente al $5 \%$ y frente al $10 \%$ : Reis $\mathrm{Pe}$ et al. ${ }^{(20)}$ realizaron un estudio tipo curva dosis-respuesta en un grupo de 25 pacientes afectados de Leucemia Mieloide Aguda en tratamiento con quimioterapia intravenosa. Aplicaron distintas concentraciones de Chamomilla Recutita en compresas húmedas a $38^{\circ} \mathrm{C}$ sobre la zona afectada, en cinco grupos. El grupo con concentraciones de 2,5\% de camomila presentó los menores tiempos de resolución, seguido de concentraciones de $5 \%$. En el grupo control sin intervención el tiempo de regresión del eritema fue mayor.

Notoginseny frente a Hirudoid $\AA$ : Gouping et al. ${ }^{(21)}$ estudiaron 65 pacientes repartidos en dos grupos. 34 fueron tratados con crema conteniendo notoginseny (preparado de medicina tradicional china que contiene cártamo, raíz de la planta de ginseng, extracto de ruibarbo y fresno espinoso) y 31 con Hirudoid $($, en ambos casos aplicados cada 4 horas, de 8 am a 8 pm hasta la desaparición de signos y síntomas. Fueron necesarias menos aplicaciones de notoginseny tanto para aliviar los signos y 
síntomas de la flebitis como para disminuir el tiempo de resolución $(p=0)$. Además resultó más efectivo en la flebitis químicas inducidas por infusiones de antibióticos, manitol y emulsiones lipídicas $(p=0)$ mientras que en la quimioterapia no hubo diferencias en ambos grupos. Notoginseng demuestra capacidad para reducir los signos y síntomas de la flebitis más rápido que el Hirudoid®.

Aloe Vera: Zheng et al. ${ }^{(28)}$ realizaron una revisión sistemática de 21 Ensayos Clínicos que estudiaron la efectividad del Aloe Vera tópico frente al no tratamiento y frente a otras sustancias tópicas como: Hirudoid®, ácido mucopolisacárido sulfúrico, $\mathrm{MgSO}_{4} \mathrm{O}$ dexametasona. Concluyen que en todos los estudios se evidencia los beneficios del aloe vera para la resolución de la flebitis así como para el alivio de los síntomas de forma significativa.

\section{Efecto de las pomadas heparinoides y geles de heparina}

Essaven Gelß frente a placebo: De Sanctis et al. ${ }^{(24)}$ compararon dos grupos de 11 y 12 pacientes respectivamente uno tratado con placebo y otro con $1 \mathrm{~g}$ de Essaven Gel (compuesto de ascinato 10mg; fosfolípidos esenciales $10 \mathrm{mg}$ y heparina sódica 10UI) una vez al día, masajeando la zona. En el grupo tratado con Essaven Gel disminuyeron tanto la temperatura de la zona $(p<0,05)$, como los síntomas mas rápido que en el grupo placebo $(p<0,05)$.

Hirudoid® frente a sulfato de magnesio: IHL NG et al. ${ }^{(26)}$ ensayaron en tres grupos de 46, 48 y 53 pacientes. Ante la aparición de enrojecimiento: en el primer grupo únicamente se retiró la vía, en el segundo se aplicó glicerina con sulfato de magnesio (GMS) y en el tercero Hirudoid® pomada durante 5 días. Se resolvió el enrojecimiento de igual forma en los tres grupos, siendo el tratado con Hirudoid® el que más tiempo tardó en recuperarse. La retirada precoz del CVP resultó igual de eficaz que la aplicación de GMS.

Gel de heparina frente placebo: Vilardell et al. ${ }^{(23)}$ llevaron a cabo un ensayo para evaluar el efecto de un gel con 1000 UI de heparina en 37 pacientes comparado con placebo en un grupo control de 40 participantes, realizándose aplicaciones 3 veces al día durante un máximo de 7 días. La curación de la flebitis fue significativamente mayor en el grupo tratado con gel de heparina $(p=0,033)$.

Gel de heparina frente a sal sódica de éster poliholósido sulfúrico: Córcoles et al. ${ }^{(27)}$ estudiaron 106 pacientes en dos grupos de 47 y 58 respectivamente, aplicando sal sódica de ester poliholósido sulfúrico al primero y heparina gel $1000 \mathrm{UI}$ al segundo. No se encontraron diferencias estadísticamente significativas en ninguno de los dos grupos en cuanto a la duración de los síntomas.

\section{Efecto de los antiinflamatorios}

Diclofenaco tópico frente a oral: Becherucci A et al. ${ }^{(22)}$ analizaron el efecto del diclofenaco tópico al 1\% aplicado 3 veces al día, frente a la administración oral de 75 mg administrados 2 veces al día y respecto a un grupo control sin tratamiento, contando con 40 pacientes en cada grupo. La intensidad de los síntomas disminuyó de forma significativa en los dos grupos de intervención para todas las variables estudiadas $(p=0,00)$, presentando efectos adversos digestivos el grupo de administración oral. Concluyen que la aplicación tópica de diclofenaco es una alternativa efectiva y segura para tratar la flebitis. 


\section{Efecto de los vasodilatadores}

Nitroglicerina (NTG) en parche frente a Pomada heparinoide: Ruiz Trillo et al. ${ }^{(25)}$ compararon dos grupos de 11 pacientes que fueron tratados: uno con parche de nitroglicerina de $5 \mathrm{mg}$ y otro con pomada heparinoide durante tres días. Con el parche de nitroglicerina desaparecieron todos los síntomas al tercer día en mayor número de pacientes que con la pomada heparinoide.

Las dos revisiones restantes no se enmarcan dentro de éstos grupos ya que los estudios que las conforman pertenecen a todos ellos.

\section{Tabla III.- Cuadro de extracción de datos}

\begin{tabular}{|c|c|c|c|}
\hline \multicolumn{4}{|c|}{ Reis PE et al / EC tipo Curva-Dosis respuesta / Brasil 2011} \\
\hline $\begin{array}{l}\text { POBLACIÓN } \\
25 \text { pacientes> } 18 \text { años: } \\
50 \% \text { mujeres, entre } 20 \\
\text { y } 30 \text { años. } \\
\text { Diagnosticados de } \\
\text { Leucemia Mieloide } \\
\text { Aguda, sometidos a } \\
\text { tratamiento } \\
\text { quimioterápico } \\
\text { Con flebitis Grado II } \\
\text { (escala INS) } \\
\text { Hospitalizados en } \\
\text { unidad de oncología } \\
\text { Criterios de exclusión: } \\
\text { reacción adversa a la } \\
\text { chamomilla, } \\
\text { prescripción de } \\
\text { tratamiento } \\
\text { antiinflamatorio } \\
\text { Evaluación diaria } 3 \\
\text { veces al día hasta } \\
\text { desaparición del } \\
\text { eritema }\end{array}$ & $\begin{array}{l}\text { INTERVENCIÓN } \\
5 \text { Grupos ( } n=5 \text { ): grupo } \\
\text { control sin tratamiento, } \\
\text { A, B, C y D aplicando } \\
\text { compresas de algodón } \\
\text { humedecidas con } \\
\text { infusión de Chamomilla } \\
\text { Recutita al } 1 \%, 2,5 \% \text {, } \\
5 \% \text { y } 10 \% \text { a } 38^{\circ} C \text {, } \\
\text { protegidas con film } \\
\text { transparente, durante } \\
20 \text { minutos, cada } 5 \\
\text { minutos,tres veces al } \\
\text { día (mañana tarde y } \\
\text { noche) } \\
\text {-Signos y síntomas de } \\
\text { flebitis: escala INS } \\
\text {-Eritema: film } \\
\text { cuadriculado en cm } \\
\text {-Tiempo de regresión } \\
\text { del eritema } \\
\text {-Toxicidad: evaluación } \\
\text { visual de síntomas } \\
\text {-Efectos adversos }\end{array}$ & $\begin{array}{l}\text { RESULTADOS } \\
\text { El grupo con } \\
\text { concentraciones de } \\
2,5 \% \text { de Chamomilla } \\
\text { presentó menores } \\
\text { tiempos de regresión } \\
\text { (29,2horas desviación } \\
\text { estándar = 8,98), } \\
\text { seguido del de } 5 \% \\
\text { (38,8horas, desviación } \\
\text { estándar = 17,47) } \\
\text { El grupo control sin } \\
\text { intervención: mayor } \\
\text { tiempo de regresión del } \\
\text { eritema } \\
\text { Eficacia de la aplicación } \\
\text { de compresas } \\
\text { templadas de } \\
\text { chamomila recutita al } \\
2,5 \% \text { que supone un } \\
\text { contenido de } \\
\text { flavonoides de } 0,04 \\
\text { mg/ml }\end{array}$ & $\begin{array}{l}\text { COMENTARIOS } \\
\text { Evidencia limitada por: } \\
\text { Asignación cuasi- } \\
\text { aleatoria } \\
\text { Tamaño de la muestra } \\
\text { pequeño }\end{array}$ \\
\hline \multicolumn{4}{|c|}{ Gouping Z et al. / EC prospectivo aleatorizado / China 2003} \\
\hline $\begin{array}{l}\text { POBLACIÓN } \\
65 \text { pacientes: } 49 \% \\
\text { mujeres, entre } 4 \text { a } 90 \\
\text { años } \\
\text { Evaluación desde el } \\
\text { inicio de la flebitis hasta } \\
\text { su desaparición }\end{array}$ & $\begin{array}{l}\text { INTERVENCIÓN } \\
\text { Grupo A: }(n=34) ; \\
\text { aplicaciones de } 14 \mathrm{~g} \\
\text { notoginseny } \\
\text { Grupo B: }(\mathrm{n}=31) ; \\
\text { aplicaciones de } \\
\text { Hirudoid® } \\
\text { En ambos casos /4h de } \\
\text { 8h am a } 8 \mathrm{~h} \text { pm } \\
\text {-Signos y síntomas de } \\
\text { flebitis: escala INS } \\
\text {-Efectos adversos }\end{array}$ & $\begin{array}{l}\text { RESULTADOS } \\
\text { En el Grupo A: } \\
\text {-Mayor eficacia para } \\
\text { aliviar signos y } \\
\text { síntomas }(p=0) \\
\text { - Mejor tiempo de } \\
\text { resolución }(p=0) \\
\text {-Mejor tiempo de } \\
\text { resolución en flebitis } \\
\text { químicas (antibióticos, } \\
\text { emulsiones lipídicas y } \\
\text { manitol) } \\
\text {-Menor coste } \\
\text { En infusiones } \\
\text { quimioterápicas no } \\
\text { diferencias en ambos } \\
\text { grupos }\end{array}$ & $\begin{array}{l}\text { COMENTARIOS } \\
\text { EI } 30 \% \text { de los pacientes } \\
\text { pediátricos } \\
\text { Dificultades en su } \\
\text { aplicabilidad por no } \\
\text { estar comercializado en } \\
\text { occidente }\end{array}$ \\
\hline
\end{tabular}




\begin{tabular}{|c|c|c|c|}
\hline \multicolumn{4}{|c|}{ Becherucci A et al. / EC prospectivo aleatorizado / Argentina 2000} \\
\hline $\begin{array}{l}\text { POBLACIÓN } \\
120 \text { pacientes > } 18 \\
\text { años: } 58 \% \text { mujeres, } \\
\text { entre } 59 \pm 16 \text { años } \\
\text { Excluidos: flebitis en } \\
\text { miembros inferiores, } \\
\text { alteraciones previas de } \\
\text { la piel, embarazo, } \\
\text { úlcera gastroduodenal, } \\
\text { colitis ulcerosa, } \\
\text { alteraciones de la } \\
\text { coagulación, } \\
\text { insuficiencia hepática o } \\
\text { renal, asma o } \\
\text { intolerancia al } \\
\text { diclofenaco } \\
\text { Hospitalizados en una } \\
\text { unidad de Medicina } \\
\text { Interna } \\
\text { Evaluación: Desde el } \\
\text { inicio de la flebitis hasta } \\
\text { las } 48 h\end{array}$ & $\begin{array}{l}\text { INTERVENCIÓN } \\
\text { Grupo control }(\mathrm{n}=40) \text { : } \\
\text { sin tratamiento } \\
\text { Grupo tópico }(\mathrm{n}=40) \text { : } \\
\text { aplicación de } \\
\text { diclofenaco } \\
\text { dietilamonico al } 1 \% \\
\text { cada 8h durante } 48 \mathrm{~h} \\
\text { Grupo oral: } \\
\text { administración de } \\
\text { diclofenaco } 75 \mathrm{mg} \text { oral } \\
\text { cada } 12 \mathrm{~h} \text { durante } 48 \mathrm{~h} \\
\text {-Rubor y tumor : cm } \\
\text {-Calor: terrmómetro de } \\
\text { contacto } \\
\text {-Dolor: escala visual } \\
\text { analógica } \\
\text {-Se consideró resultado } \\
\text { positivo la disminución } \\
\text { de la intensidad de los } \\
\text { síntomas del } 30 \% \\
\text {-Efectos adversos }\end{array}$ & $\begin{array}{l}\text { RESULTADOS } \\
\text { La intensidad de los } \\
\text { síntomas disminuyó } \\
\text { significativamente en } \\
\text { los dos grupos de } \\
\text { intervención para todas } \\
\text { las variables } \\
\text { estudiadas: rubor } \\
\text { ( } p=0,00) \text {, tumor }(p=0,00) \\
\text { calor }(p=0,00), \text { dolor } \\
\text { ( } p=0,00) \\
\text {-Los efectos adversos } \\
\text { digestivos fueron } \\
\text { mayores en el grupo } \\
\text { que recibió el fármaco } \\
\text { oral } \\
\text { - Eficacia del } \\
\text { diclofenaco tanto tópico } \\
\text { como oral } \\
\text { Mayor seguridad en la } \\
\text { aplicación tópica }\end{array}$ & $\begin{array}{l}\text { COMENTARIOS } \\
\text { Nivel de evidencia 1c } \\
\text { Grado de } \\
\text { recomendación } \\
\text { moderado que sugiere } \\
\text { su aplicación en la } \\
\text { práctica clínica }\end{array}$ \\
\hline \multicolumn{4}{|c|}{ Vilardell M et al. /EC Prospectivo Aleatorizado / España 1999} \\
\hline $\begin{array}{l}\text { POBLACIÓN } \\
132 \text { pacientes > de } 18 \\
\text { años: } 39 \% \text { mujeres } \\
\text { (55 pacientes se } \\
\text { perdieron) } \\
\text { Hospitalizados en una } \\
\text { unidad de Medicina } \\
\text { Interna } \\
\text { Evaluación cada } 24 \\
\text { horas durante } 7 \text { días } \\
\text { máximo }\end{array}$ & $\begin{array}{l}\text { INTERVENCIÓN } \\
\text { Grupo control ( } \mathrm{n}=40 \text { ): } \\
\text { Placebo } \\
\text { Grupo de intervención } \\
\text { ( } \mathrm{n}=37) \text { : Gel tópico con } \\
1000 \text { UI de heparina. } \\
\text { Aplicado } 3 \text { veces /día } \\
\text { durante } 7 \text { días máximo } \\
\text { en ambos grupos. } \\
\text { Se administraron } \\
\text { anticoagulantes a } 7 \\
\text { participantes, } 6 \text { en el } \\
\text { grupo de intervención y } \\
1 \text { del grupo control } \\
\text {-Signos y síntomas de } \\
\text { flebitis: no síntomas, } \\
\text { leve (induración, rojez o } \\
\text { temperatura local), } \\
\text { moderada (+ } \\
\text { sensibilidad) y severa } \\
\text { (+ dolor) } \\
\text {-Lesión de la vena en } \\
\text { cm } \\
\text {-Efectos adversos }\end{array}$ & $\begin{array}{l}\text { RESULTADOS } \\
\text { La curación de la flebitis } \\
\text { fue del } 44,3 \% \text { en el } \\
\text { grupo de intervención } \\
\text { vs el } 26,1 \% \text { en el grupo } \\
\text { control }(p=0,033) \\
\text { Eficacia de gel de } \\
\text { heparina ( } 1000 \text { Ul) para } \\
\text { resolver los síntomas } \\
\text { de la flebitis }\end{array}$ & $\begin{array}{l}\text { COMENTARIOS } \\
\text { Nivel de evidencia 1c } \\
\text { Grado de } \\
\text { recomendación } \\
\text { moderado que sugiere } \\
\text { su aplicación en la } \\
\text { práctica clínica } \\
\text { Doble ciego }\end{array}$ \\
\hline \multicolumn{4}{|c|}{ De Sanctis MT et al. / EC prospectivo aleatorizado / Italia y UK 2001} \\
\hline $\begin{array}{l}\text { POBLACIÓN } \\
23 \text { pacientes > } 18 \text { años: } \\
52 \% \text { mujeres, entre } \\
58,5 \pm 6,5 \text { años } \\
\text { Evaluación cada } \\
\text { semana durante } 4 \\
\text { semanas }\end{array}$ & $\begin{array}{l}\text { INTERVENCIÓN } \\
\text { Grupo placebo }(\mathrm{n}=11) \\
\text { Grupo tratamiento } \\
(\mathrm{n}=12): 1 \mathrm{~g} \text { de Essaven } \\
\text { gel@B a } 22^{\circ} \\
\text { masajeando (no informa } \\
\text { de frecuencia de } \\
\text { aplicaciones) } \\
\text { Pacientes en } \\
\text { tratamiento con } \\
\text { heparina sódica } 1 \\
\text { vez/día } \\
\text {-Temperatura de la piel: } \\
\text { (patterson digital } \\
\text { termometer) }\end{array}$ & $\begin{array}{l}\text { RESULTADOS } \\
\text { En los dos grupos } \\
\text { disminuyó la } \\
\text { temperatura a las } 4 \\
\text { semanas } \\
\text { En el grupo de Essaven } \\
\text { Gel disminuyó la } \\
\text { temperatura mas rápido } \\
(p<0,05) \text { así como los } \\
\text { síntomas }(p<0,05)\end{array}$ & $\begin{array}{l}\text { COMENTARIOS } \\
\text { Doble ciego } \\
\text { Evidencia limitada por } \\
\text { pequeño tamaño } \\
\text { muestral }\end{array}$ \\
\hline
\end{tabular}




\begin{tabular}{|c|c|c|c|}
\hline & $\begin{array}{l}\text {-Dolor, disconfor, } \\
\text { hinchazón: Escala } \\
\text { analógica de síntomas } \\
\text {-Intolerancia y efectos } \\
\text { adversos }\end{array}$ & & \\
\hline \multicolumn{4}{|c|}{ Ruiz Trillo C et al. / EC prospectivo aleatorizado / España 2006} \\
\hline $\begin{array}{l}\text { POBLACIÓN } \\
22 \text { pacientes > } 18 \text { años: } \\
68 \% \text { mujeres, entre } 18 \\
\text { y } 88 \text { años } \\
\text { Hospitalizados en una } \\
\text { Unidad de Medicina } \\
\text { Interna } \\
\text { Evaluación desde el } \\
\text { diagnóstico de la flebitis } \\
\text { hasta } 3 \text { días post } \\
\text { tratamiento }\end{array}$ & $\begin{array}{l}\text { INTERVENCIÓN } \\
\text { Grupo1 ( } \mathrm{n}=11) \text { : pomada } \\
\text { heparinoide } \\
\text { Grupo } 2(\mathrm{n}=11) \text { parche } \\
\text { transdérmico de } \\
\text { nitroglicerina (5mg) } \\
\text { - Grado de flebitis: } \\
\text { escala de Maddox } \\
\text {-Localización, movilidad } \\
\text { de la vía } \\
\text {-Sustancias perfundidas } \\
\text {-Efectos secundarios }\end{array}$ & $\begin{array}{l}\text { RESULTADOS } \\
\text { En el Grupo } 2 \\
\text { desaparecieron todos } \\
\text { los síntomas al tercer } \\
\text { día de tratamiento en } 9 \\
\text { de los } 11 \text { pacientes, } \\
\text { frente al Grupo } 1 \text { que } \\
\text { desaparecieron solo en } \\
2 \text { de los } 11 \text { pacientes. } \\
\text { El parche de } \\
\text { nitroglicerina resultó } \\
\text { mas eficaz de que la } \\
\text { pomada heparinoide }\end{array}$ & $\begin{array}{l}\text { COMENTARIOS } \\
\text { Evidencia limitada por: } \\
\text { Asignación Cuasi- } \\
\text { aleatoria } \\
\text { tamaño muestral } \\
\text { pequeño }\end{array}$ \\
\hline \multicolumn{4}{|c|}{ Ng IHL et al. / EC prospectivo aleatorizado / Singapur 2010} \\
\hline $\begin{array}{l}\text { PÖLACIÓN } \\
147 \text { pacientes > } 18 \\
\text { años } \\
\text { Excluidos los que } \\
\text { sufren trombosis, } \\
\text { afecciones de la piel o } \\
\text { presentan pus en la } \\
\text { zona a estudiar } \\
\text { Evaluación: } 5 \text { días }\end{array}$ & $\begin{array}{l}\text { INTERVENCIÓN } \\
46 \text { pacientes: cambio } \\
\text { de catéter al iniciarse la } \\
\text { rojez } \\
48 \text { pacientes: aplicación } \\
\text { de sulfato de magnesio } \\
\text { con glicerina (GMS) } \\
53 \text { pacientes: aplicación } \\
\text { de Hirudoid® } \\
\text { No se especifica la } \\
\text { frecuencia de las } \\
\text { aplicaciones } \\
\text {-Grado de rojez } \\
\text {-Tiempo en resolverse } \\
\text { la rojez }\end{array}$ & $\begin{array}{l}\text { RESULTADOS } \\
\text { En los tres grupos se } \\
\text { resuelve la rojez por } \\
\text { igual sin diferencias } \\
\text { significativas. } \\
\text { Cambio de catéter vs } \\
\text { Hirudoid®: con } \\
\text { Hirudoid® se prolongó } \\
\text { el tiempo de resolución } \\
\text { (p<0,05) } \\
\text { Cambio de catéter vs } \\
\text { GMS: no diferencias. } \\
\text { Para resolver el eritema } \\
\text { o flebitis precoz basta } \\
\text { con retirada del catéter } \\
\text { de forma temprana. }\end{array}$ & $\begin{array}{l}\text { COMENTARIOS } \\
\text { Nivel de evidencia 1c } \\
\text { Grado de } \\
\text { recomendación } \\
\text { moderado que sugiere } \\
\text { su aplicación en la } \\
\text { práctica clínica }\end{array}$ \\
\hline \multicolumn{4}{|c|}{ Córcoles MP et al. EC prospectivo aleatorizado / España 1996} \\
\hline $\begin{array}{l}\text { POBLACIÓN } \\
106 \text { pacientes > } 18 \\
\text { años: } 30 \% \text { mujeres } \\
\text { entre } 64,93 \pm 17,52 \\
\text { Hospitalizados en una } \\
\text { unidad de Cirugía } \\
\text { General }\end{array}$ & $\begin{array}{l}\text { INTERVENCIÓN } \\
47 \text { pacientes: sal sódica } \\
\text { de ester poliholosido } \\
\text { sulfúrico tópica } \\
58 \text { pacientes: heparina } \\
\text { 1000U.I gel } \\
\text {-Tumoración de la } \\
\text { flebitis; en cm con cinta } \\
\text { métrica } \\
\text {-Dolor, calor, rubor, (no } \\
\text { se especifica en qué } \\
\text { escala de medida) } \\
\text {-Tiempo de duración de } \\
\text { la sintomatología }\end{array}$ & $\begin{array}{l}\text { RESULTADOS } \\
\text { No se encuentran } \\
\text { diferencias } \\
\text { estadísticamente } \\
\text { significativas de un } \\
\text { tratamiento frente al } \\
\text { otro respecto a la } \\
\text { duración de los } \\
\text { síntomas. }\end{array}$ & $\begin{array}{l}\text { COMENTARIOS } \\
\text { Evidencia limitada por } \\
\text { Asignación cuasi- } \\
\text { aleatoria } \\
\text { No ha tenido en cuenta } \\
\text { posibles efectos } \\
\text { adversos }\end{array}$ \\
\hline \multicolumn{4}{|c|}{ Zheng GH et al. / Revisión sistemática / Colaboración Cochrane / China 2014} \\
\hline $\begin{array}{l}\text { POBLACIÓN } \\
\text { Analizan } 43 \text { EC: } 35 \\
\text { aleatorios y } 8 \\
\text { cuasialeatorios. } \\
21 \text { EC con } 1919 \\
\text { participantes abordan el } \\
\text { tratamiento de la flebitis } \\
\text { Criterios de inclusión: } \\
\text { Flebitis asociada a un } \\
\text { dispositivo venoso } \\
\text { periférico en brazos o } \\
\text { dorso del pié }\end{array}$ & $\begin{array}{l}\text { INTERVENCIÓN } \\
\text { Aplicación externa de } \\
\text { aloe vera fresco sólo o } \\
\text { con otros productos: } \\
\text { compresas húmeda de } \\
75 \% \text { de alcohol o 33\%, } \\
50 \% \text { o } 75 \% \text { MgSO4, } \\
\text { frente a: } \\
\text {-No tratamiento } \\
\text { - Tratamientos de } \\
\text { rutina: Hirudoid } \mathbb{B}, \\
\text { mocopolisacáricos, }\end{array}$ & $\begin{array}{l}\text { RESULTADOS } \\
\text { Aloe vera sólo o en } \\
\text { combinación, es más } \\
\text { efectivo que el no } \\
\text { tratamiento y que la } \\
\text { aplicación de } \\
\text { tratamiento de rutina. } \\
\text { Mejoran lo síntomas de } \\
\text { flebitis tales como } \\
\text { tiempo de reducción del } \\
\text { eritema, dolor en el } \\
\text { punto de punción y } \\
\text { tiempo de resolución de }\end{array}$ & $\begin{array}{l}\text { COMENTARIOS } \\
\text { Evidencia limitada por } \\
\text { la pobre calidad } \\
\text { metodológica de los } \\
\text { estudios y por la } \\
\text { variación en la dirección } \\
\text { y número de efectos } \\
\text { extraídos de los mismos }\end{array}$ \\
\hline
\end{tabular}




\begin{tabular}{|c|c|c|c|}
\hline $\begin{array}{l}\text { Evaluación de } 1 \text { a } 15 \\
\text { días }\end{array}$ & $\begin{array}{l}\text { ácido sulfonico y } \\
\text { dexametasona } \\
\text { Medición de resultados } \\
\text { Primario: Tasa de } \\
\text { mejoría y resolución de } \\
\text { flebitis. } \\
\text { Secundarios: mejoría } \\
\text { de los síntomas de } \\
\text { flebitis }\end{array}$ & la flebitis & \\
\hline \multicolumn{4}{|c|}{ Dos Reis PE et al. / Revisión sistemática / Brasil 2009} \\
\hline $\begin{array}{l}\text { POBLACIÓN } \\
\text { Analizan: } 4 \text { ECA y } 1 \\
\text { estudio cuasialeatorio } \\
\text { con } 375 \text { participantes } \\
\text { Criterios de inclusión: } \\
\text { Flebitis derivada de la } \\
\text { terapia intravenosa } \\
\text { periférica }\end{array}$ & $\begin{array}{l}\text { INTERVENCIÓN } \\
\text { Aplicación de: } \\
\text { nitroglicerina (NTG), } \\
\text { parche transdérmico o } \\
\text { gel, cremas de heparina } \\
\text { o heparinoides, } \\
\text { pyroxicam en gel, } \\
\text { notoginseny crema y } \\
\text { diclofenaco en gel y } \\
\text { oral } \\
\text { Las variables: dolor, } \\
\text { edema, eritema, } \\
\text { grado/área de flebitis, } \\
\text { tiempo de regresión de } \\
\text { la flebitis }\end{array}$ & $\begin{array}{l}\text { RESULTADOS } \\
\text { Notoginseny crema y } \\
\text { nitroglicerina reducen } \\
\text { el proceso inflamatorio } \\
\text { de forma efectiva frente } \\
\text { a sustancias } \\
\text { heparinoides. } \\
\text { Las sustancias } \\
\text { heparinoides son tan } \\
\text { eficaces como los } \\
\text { antiinflamatorios tópicos }\end{array}$ & $\begin{array}{l}\text { COMENTARIOS } \\
\text { Evidencia limitada en } \\
\text { cuanto a que el } \\
\text { notoginseny y la NTG } \\
\text { aplicados de forma } \\
\text { tópica sean el } \\
\text { tratamiento de elección, } \\
\text { debido a las } \\
\text { limitaciones } \\
\text { metodológicas que } \\
\text { presentan los estudios. }\end{array}$ \\
\hline \multicolumn{4}{|c|}{ Di Nisio M et al. / Revisión Sistemática / Italia 2015} \\
\hline $\begin{array}{l}\text { POBLACIÓN } \\
\text { Analizaron: } 13 \text { ensayos } \\
\text { con } 917 \text { participantes; } \\
11 \text { evaluaron } \\
\text { tratamiento tópico, } 2 \\
\text { tratamiento oral y } 2 \\
\text { parenteral. } \\
\text { Criterios de inclusión: } \\
\text { Tromboflebitis } \\
\text { superficial del miembro } \\
\text { superior relacionada } \\
\text { con la terapia de } \\
\text { infusión }\end{array}$ & $\begin{array}{l}\text { INTERVENCIÓN } \\
\text { Tratamiento: Cualquier } \\
\text { medicación tópica } \\
\text { (Essaven®, Phebolan®, } \\
\text { diclofenaco,NTG, } \\
\text { notoginseny y } \\
\text { mucopolisacáridos) oral o } \\
\text { parenteral para aliviar los } \\
\text { síntomas y signos o evitar } \\
\text { complicaciones frente } \\
\text { a placebo no intervención } \\
\text { Medición de resultados } \\
\text { Primarios: Reducción o } \\
\text { resolución signos y } \\
\text { síntomas: dolor, } \\
\text { induración, eritema. } \\
\text { Secundarios: } \\
\text { Extensión de } \\
\text { tromboflebitis, } \\
\text { supuración y sepsis } \\
\text { relacionada con catéter }\end{array}$ & $\begin{array}{l}\text { RESULTADOS } \\
\text { Los geles heparinoides } \\
\text { o el diclofenaco tópico } \\
\text { reducen } \\
\text { significativamente la } \\
\text { intensidad de los } \\
\text { síntomas y signos } \\
\text { clínicos y consiguen } \\
\text { una resolución } \\
\text { completa frente a } \\
\text { placebo o la no } \\
\text { intervención. } \\
\text { Ningún estudió informó } \\
\text { de supuración, ni sepsis } \\
\text { relacionadas con } \\
\text { catéter }\end{array}$ & $\begin{array}{l}\text { COMENTARIOS } \\
\text { Evidencia limitada. Los } \\
\text { datos parecen } \\
\text { demasiado preliminares } \\
\text { para valorar la } \\
\text { efectividad y seguridad } \\
\text { de los tratamientos } \\
\text { tópicos analizados. }\end{array}$ \\
\hline
\end{tabular}

\section{DISCUSIÓN}

Constituyendo el tema que nos ocupa un problema tan relevante en la práctica enfermera tanto per se, como por las consecuencias que de él se derivan, llama la atención la escasez de estudios encontrados que lo abordan.

Principales resultados: Después del análisis pormenorizado de todos los estudios, se constata que los tratamientos tópicos de aloe vera, Chamomilla Recutita en compresas húmedas a $38^{\circ} \mathrm{C}$ sobre la zona afectada y diclofenaco tópico son efectivos frente a la no intervención. Así como el gel de heparina y Essaven Gelß frente a placebo. Otros productos tópicos como notoginseny y parche de nitroglicerina $5 \mathrm{mg}$, aceleran la mejoría de los síntomas de la flebitis comparados con sustancias heparinoides (Hirudoid®). Además la retirada del catéter de forma temprana, como 
única medida, frente a la aplicación de sulfato de magnesio con glicerina y frente Hirudoid®, resulta suficiente para resolver el eritema. Se muestran idénticos resultados al comparar sal sódica de ester poliholósido sulfúrico y heparina gel 1000U.I.

Sesgos de los EC: A pesar de presentar una calidad metodológica calificada $\geq 7$ por el CASPe, algunos estudios presentan sesgos considerables: en tres de ellos ${ }^{(20,24,25)}$ la muestra no supera los 25 sujetos, dando lugar a grupos control y experimental muy reducidos, lo que puede provocar estimaciones sesgadas de los efectos del tratamiento a pesar de las correcciones estadísticas. Dos estudios ${ }^{(20,27)}$ presentan una distribución de la población cuasialeatoria y solo dos $\mathrm{EC}^{(23,24)}$ presentan doble ciego.

Limitaciones: Las limitaciones de la revisión sistemática se enmarcan en el ámbito de la búsquedas en las bases de datos señaladas, a través de la Biblioteca Sanitaria online de Castilla y León, en inglés y en español. El hecho de que se haya ampliado la búsqueda a los últimos 20 años responde a la escasez de EC recientes relativos al tratamiento tópico, lo que puede provocar que los resultados no se adapten a la realidad actual.

Acuerdos y desacuerdos con otros revisores: En cuanto a las revisiones analizadas: Dos Reis et $\mathrm{al}^{(29)}$ analizan 5 EC concluyendo que el notoginseny y la nitroglicerina son los productos más efectivos para tratar la flebitis aunque muestran un nivel de evidencia limitada. Di Nisio et $a^{(30)}$ evalúan 13 estudios en los que se contrastan distintas intervenciones para el tratamiento de la tromboflebitis, presentan resultados similares a los de esta revisión afirmando que los geles heparinoides y los antiinflamatorios tópicos muestran efectividad aunque con reservas a la espera de estudios más concluyentes. No obstante, presentan diferencias importantes: realizan comparativas con tratamientos no sólo tópicos, también orales e intravenosos, además solo 5 de los $13 \mathrm{EC}$ que analizan están realizados dentro de la franja de los últimos 20 años, el resto son anteriores, consideramos que EC tan antiguos puedan alejarse demasiado de las prácticas de inserción de catéteres en la actualidad y aborda la flebitis causada por trombo, aspecto que no es objeto de este estudio.

Implicaciones para la práctica clínica: No todos los resultados con significación estadística son clínicamente relevantes o muestran un nivel de evidencia, según criterios de The Johanna Briggs Institute ${ }^{(19)}$, que permitan su recomendación en la práctica clínica. El notoginseny, el diclofenaco tópico y heparina gel 100Ul. muestran un nivel de evidencia 1c y un grado de recomendación moderado que sugiere que se considere su aplicabilidad en la práctica clínica. Sin embargo hay que puntualizar que el notoginseng es un producto de la industria farmacéutica china (departamento del Hospital SunYat-Sen University) que no está comercializado en España como tal, por tanto se precisarían estudios que puedan demostrar la verdadera eficacia del notoginseny y sus componentes. El diclofenaco es un antiinflamatorio utilizado en diversas patologías y la heparina gel es el único compuesto que según la Agencia Española del Medicamento tiene entre sus indicaciones el tratamiento de la flebitis postcateterización. Por otro lado, el aloe vera también presenta un nivel de evidencia 1c y es un producto de uso generalizado en la medicina china, con gran potencial y que puede ser útil para el desarrollo de compuestos derivados del mismo y su utilización en Occidente. 


\section{CONCLUSIONES}

La evidencia para el tratamiento de la flebitis secundaria a cateterización venosa periférica con productos de aplicación externa está limitada por la baja calidad metodológica de los estudios evaluados. Los efectos positivos observados en los ensayos clínicos con el gel de heparina y la revisión sobre la aplicación de aloe vera deben valorarse con reservas: no obstante son dos productos merecedores de especial atención (principalmente el aloe vera) y dignos de consideración para su posible aplicabilidad en el futuro. De todo ello se desprende la necesidad de generar investigación primaria, con ensayos clínicos más consistentes que incluyan muestras más grandes y un mejor diseño que tenga en cuenta una adecuada valoración de los potenciales efectos secundarios.

\section{REFERENCIAS}

1.- Rojas-Sánchez L Z, Parra D I, Camargo-Figuera F A. Incidencia y factores asociados al desarrollo de flebitis: resultados del estudio piloto de una cohorte. Referencia 2015;IV(4):61-67, http://dx.doi.org/10.12707/RIII13141

2.- Mestre Roca G, Berbel Bertolo C, Tortajada Lopez P, Gallemi Samaranch G, Aguilar Ramírez M A, Caylà Buqueras J, et al. Assessing the influence of risk factors on rates and dynamics of peripheral vein phlebitis: An observational cohort study. Med Clin (Barc). 2012;139(5):185-191, http://dx.doi.org/10.1016/j.medcli.2011.12.021

3.- Cicolini G, Manzoli L, Simonetti V, Flacco ME, Comparcini D, Capasso L, et al. Phlebitis risk varies by peripheral venous catéter site and increases after 96 hours: a large multi-centre prospective study. J Adv Nurs. 2014 Nov;70(11):2539-49, http://dx.doi.org/10.1111/jan.12403

4. Martínez JA, Piazuelo $M$, Almela $M$, Blecua $P$, Gallardo $R$, Rodríguez $S$, et al. Evaluation of add-on devices for the prevention of phlebitis and other complications associated with the use of peripheral catheters in hospitalised adults: a randomised controlled study. J Hosp Infect. 2009 Oct;73(2):135-42, http://dx.doi.org/10.1016/j.jhin.2009.06.031

5.- Hadaway L. Short peripheral intravenous catheters and infections. J Infus Nurs. 2012 Jul-Aug;35(4):230-40. DOI: 10.1097/NAN.0b013e31825af099.

6.- Marsh N, Mihala G, Ray-Barruel $\mathrm{G}^{2}$, Webster J, Wallis MC, Rickard CM. Inter-rater agreement on PIVC associated phlebitis signs, symtoms and scales. J Eval Clin Pract. 2015 Oct;21(5):893-9, http://dx.doi.org/10.1111/jep.12396

7.- Woody G, Davis BA. Increasing nurse competence in peripheral intravenous therapy. J Infus Nurs. 2013 Nov-Dec;36(6):413-9. DOI: 10.1097/NAN.0000000000000013.

8.- Ferrer $C$, Almirante $B$. Venous catheter-related infections. Enferm Infecc Microbiol Clin. 2014 Feb;32(2):115-24, http://dx.doi.org/10.1016/j.eimc.2013.12.002

9.- González López JL, Arribi Vilela A, Fernández del Palacio E, Olivares Corral J, Benedicto Martí C, Herrera Portal P. Indwell times, complications and costs of open vs closed safety peripheral intravenous catheters: a randomized study. J Hosp Infect. 2014 Feb;86(2):117-26, http://dx.doi.org/10.1016/j.jhin.2013.10.008

10.- Stuart RL, Cameron DR, Scott C, Kotsanas D, Grayson ML, Korman TM, et al. Peripheral intravenous catheter-associated Staphylococcus aureus bacteraemia: more than 5 years of prospective data from two tertiary health services. Med J Aust. 2013 Jun 3;198(10):551-3, http://dx.doi.org/10.5694/mja12.11699

11.- Ferrete-Morales $C$, Vázquez-Pérez MA, Sánchez-Berna $M$, Gilabert-Cerrol, Corzo-Delgado JE, Pineda-Vergara JA, et al. Incidence of phlebitis due to peripherally 
inserted venous catheters: impact of a catheter management protocol. Enferm Clin. 2010 Jan-Feb;20(1):3-9, http://dx.doi.org/10.1016/j.enfcli.2009.10.001

12.- Bulechek GM, Butcher HK, Dochterman JM, editoras. Clasificación de intervenciones de enfermería (NIC). 5ª ed. Madrid: Elsevier Mostby; 2013, p. 764.

13.- Smith SE, Duell DJ, Martin BC. Técnicas de enfermería clínica. De las técnicas básicas a las avanzadas. Vol II. 7ª ed. Madrid: Pearson; 2009, p. 1097.

14.- Alexander M, Corrigan A, Gorskil L, Hankins J, Perucca R. Infusion nursing and evidence-based approach. $3^{a}$ ed. St Luis, Missouri: Saunder Elsevier, 2010, p. 475.

15.- Ingram P, Lavery I. Peripheral intravenous therapy: key risks and implications for practice. Nurs Stand. 2005;19(46):55-64.

16.- Tjon JA, Ansani NT. Transdermal nitroglycerin for the prevention of intravenous infusion failure due to phlebitis and extravasation. Ann Pharmacother. 2000;34(10):1189-1192

17.- Grupo de trabajo de la Guía de Práctica Clínica sobre Terapia Intravenosa con Dispositivos no Permanentes en Adultos. Guía de Práctica Clínica sobre Terapia Intravenosa con Dispositivos no Permanentes en Adultos. Ministerio de Sanidad, Servicios Sociales e Igualdad. Agencia de Evaluación de Tecnologías Sanitarias de Andalucía (AETSA); 2014. Guías de Práctica Clínica en el SNS.

18.- Guías CASPe de Lectura Crítica de la Literatura Médica CASPe 2014. [consulta 11 En 2016]. Disponible en: http://www.redcaspe.org.

19.- Joanna Briggs Institute JBI CONNECT Espa na [Internet].Madrid: Centre col.laborador espanyol del JBI; 20014 [consulta 11 En 2016]. Disponible en: http://joannabriggs.org/jbi-approach.html\#tabbed-nav=Grades-of-Recommendation.

20.- Reis PED Dos, Carvalho EC de, Bueno PCP, Bastos JK. Clinical application of Chamomilla recutita in phlebitis: dose response curve study. Rev Lat Am Enfermagem. $2011 ; 19$ (1):3-10. DOI: 10.1590/S0104-11692011000100002

21.- Gouping Z, Wan-Er T, Xue-Ling W, Min-Qian X, Kun F, Turale S, et al. Notoginseny cream in the treatment of phlebitis. J Infus Nurs. $2003 ; 26$ (1):49-54. DOI: 10.1097/00129804-200301000-00007

22.- Becherucci A, Bagilet D, Marenghini J, Diab M, Biancardi H. [Effect of topical and oral diclofenac on superficial thrombophlebitis caused by intravenous infusion]. Med Clin (Barc). 2000 Mar 18;114(10):371-3. DOI:10.1016/S0025-7753(00)71300-5

23.- Vilardell M, Sabat $D$, Arnaiz JA, Bleda MJ, Castel JM, Laporte JR, Vallvé C. Topical heparin for the treatment of acute superficial phlebitis secondary to indwelling intravenous catheter. A double-blind, randomized, placebo-controlled trial. Eur J Clin Pharmacol. 1999 Feb;54(12):917-21. DOI: 10.1007/s002280050575

24.- De Sanctis MT, Cesarone MR, Incandela L, Belcaro G, Griffin M. Treatment of superficial vein thrombophlebitis of the arm with Essaven gel--a placebo-controlled, randomized study. Angiology. 2001 Dec;52 Suppl 3:S63-7. DOI: 10.1177/000331970105200312.

25.- Ruiz Trillo C, Borrero Esteban MP. Tratamientos aplicados a la Flebitis posperfusión. Rev ROL Enferm.2006; 29(2): 55-60.

26.- $\mathrm{Ng} \mathrm{IHL}$, Jabin K. Best Treatment Modality for Superficial Phlebitis. Ann Acad

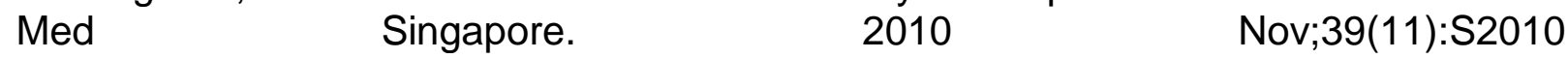
http://www.annals.edu.sg/pdf/39VolNo11SuppINov2010/V39N11\%28Suppl\%29.pdf

27.- Córcoles MP, Ruiz T, García D. Flebitis postpunción. Rev ROL Enferm.1996 Sept; 217:13-16

28.- Zheng GH, Yang L, Chen HY, Chu JF, Mei L. Aloe vera for prevention and treatment of infusion phlebitis. Cochrane Database Syst Rev 2014; 6: CD009162. DOI:10.1002/14651858.CD009162.pub2. 
29.- Reis PED Dos, Silveira RC, Vasques $\mathrm{Cl}$, Carvalho EC de. Pharmacological interventions to treat phlebitis: systematic review. J Infus Nurs. 2009; 32(2): 74-9. DOI:10.1097/NAN.0b013e318198d497.

30.- Di Nisio M, Peinemann F, Porreca E, Rutjes AW. Treatment for superficial infusion thrombophlebitis of the upper extremity. Cochrane Database Syst Rev. 2015 Nov 20;11:CD011015. DOI: 10.1002/14651858.CD011015.pub2.

Recibido: 05 de junio 2016;

Aceptado: 30 de agosto 2016 